\title{
Efficacy of pediculicides used in Sri Lanka: comparison of Western, alternative medicinal and home-made herbal preparations
}

\author{
H Banneheke ${ }^{1}$, V Jayasuriya ${ }^{2}$ \\ Ceylon Medical Journal 2017; 62:199-202 \\ DOI: http://doi.org/10.4038/cmj.v62i3.8525
}

\begin{abstract}
Human pediculosis of scalp is caused by an ectoparasite Pediculus humanus capitis. It is easily transmitted by contact, commonly among school children, causing scalp itching, poor sleep, excoriations, lymphadenopathy, as well as social stigma, embarrassment, and low self-esteem [1].
\end{abstract}

There are several treatment modalities such as, removal of nits and adults, application of homemade herbal remedies and applications of commercial and medicinal preparations which are easily accessible as over-the-counter products. There is evidence of treatment failure due to drug resistance [2]. In Sri Lanka, permethrin is the most widely used commercially available chemical pediculicide. Lindane (gamma-hexa chloro cyclo hexane $/ \gamma-\mathrm{HCH}$ ) used previously in such products, was prohibited in 2012, after it was confirmed to be associated with non-Hodgkin's lymphoma [3]. Alternative medicinal products labeled as pediculicides are also commercially available. Unlike Western medical products which are classified as drugs, alternative medicinal products labeled as cosmetics or alternative medicines could bypass safety testing and registration requirements. The paper presents the findings of a study comparing the efficacy of five pediculicide which include both commercial and herbal preparations used in Sri Lanka. alternative medicinal pediculicide, and two herbal preparation extracts of 'Godapara'(Dilleniaretusa) and 'Veralu'/Ceylon olive leaves (Elaeocarpus serratus) were tested in vitro for effectiveness using live head lice and nits obtained from 35 school children with scalp pediculosis. Permission was obtained from the Ethics Review Committee of the Faculty of Medical Sciences, University of Sri Jayewardenepura. Parents of grade 1-9 children from schools in the Western Province were contacted through their children. After providing information about the study and obtaining written consent from parents, they were provided with instructions and containers for collection of samples at home. Parents were advised to gather at least 15 lice and 10 strands of hair containing nits from the child and hand it over to the research team outside the school. This safeguarded against children being identified by peers and any risk of stigmatization. All the classes with participating children received information about safe remedies for treatment of head lice to avoid identifying those that were affected. The samples were delivered within two hours of collection to the laboratory and the experiment was commenced immediately upon arrival.

As recommended in previous studies, the viable lice in the samples were pooled into one collection as both resistant and sensitive lice can be found on the

\footnotetext{
${ }^{I}$ Department of Parasitology, Faculty of Medical Sciences, University of Sri Jayewardenepura and ${ }^{2}$ Yeates School of Graduate Studies, Ryerson University, Toronto, Canada.

Correspondence: HB, E-mail: <hasini@sjp.ac.lk>. Received 10 June 2017 and revised version accepted 24 July 2017.
}

Two chemical pediculicides $0.5 \%$ permethrine and gamma-hexa chloro cyclo hexane (lindane), one 
same person's head from prior exposure to pediculicides [2].

Permethrine, lindane, and the alternative medicinal pediculicide were used in its original form and plant extracts were made manually by grinding $250 \mathrm{~g}$ of the plant without adding water or any other liquid. Cotton cloth disks $(5 \mathrm{~cm}$ diameter) were placed in six petri dishes and five of them were soaked with $1 \mathrm{ml}$ of pediculicide and the sixth with distilled water as a control. After observing morphological features for confirmation using a dissecting microscope, a batch of 15 live head lice were placed on each soaked cotton disk. The lice were examined for viability at 5, 10, 20, 30 minutes and 1, 2, 3, 6 hours. Death of lice was confirmed by the absence of movement and loss of gut peristalsis when viewed under a microscope. The test was repeated six times for each substance.

The efficacy of Western medicinal pediculicides (permethrin and lindane) and the alternative medicinal pediculicide against nits (ovicidal activity) was also tested. Herbal preparations were not included due to a nonscientific reason. Snips of hair containing viable nits from different participants were mixed to create bundles $5 \mathrm{~cm}$ in length, each with 10 viable nits. Each bundle was immersed in $1 \mathrm{ml}$ of pediculicide for the recommended duration given in the patient information leaflet of each product. Subsequently they were immersed in a non-medicated bath shampoo, washed with water, and examined daily for hatching using a dissecting microscope. Distilled water was used as the wet control. Another set of hair was kept without water or pediculicides as a dry control. The test was repeated four times.

Mass spectrometry was used in an attempt to identify the chemical compounds of the alternative medicinal pediculicides. However, this was unsuccessful due to the extremely oily nature of the compound.

Figure 1 shows the proportion of lice and nymphs killed by each substance over time, an average of the six samples were tested for each. Five minutes after application, Godapara and Ceylon olive (Veralu) were the most effective, killing 62\%-63\% compared to $37 \%$ for permethrin and $14 \%$ for alternative medicinal pediculicide $(p<0.05)$. Lindane was the least effective at one hour, and remained so until 3 hours after application. The alternative medicinal pediculicide, although less effective at 5 and 10 minutes, showed the highest proportion of head lice killed at 20 minutes $(81 \%)$. At 3 hours, the alternative medicinal pediculicide showed $100 \%$ mortality. Godapara and Ceylon olive (Veralu) efficacy was around $70 \%-80 \%$ throughout the 6 hours. Godapara (78.9\%), Veralu (74.4\%), and permethrin $(72.5 \%)$ also showed significantly higher efficacy compared to lindane $40.4 \%$ at 6 hours $(p<0.01)$.

The results of the efficacy of Western medicinal pediculicides (permethrin and lindane) and the alternative medicinal pediculicides against nits (ovicidal activity) are presented in table 1 . None of the nits exposed to the alternative medicinal pediculicides hatched across the four tests showing a $100 \%$ ovicidal effect. Lindane proved to be less ovicidal than permethrin in this experiment. Overall, across the in-vitro experiments the alternative medicinal pediculicide appeared to show a higher efficacy than the commercially available Western medicinal pediculicides.

There are two important findings from this study. The first, and the more alarming one, is the strength of the alternative medicinal pediculicide, a product which does not contain any information about the nature or the strength of the active ingredients. Although the recommended contact time for this product was 8 hours, it killed more than $80 \%$ of adult lice within 20 minutes, much more so than the permitted permethrin $0.5 \%$ which needed 3-6 hours exposure to show as much efficacy. Although herbal preparations also showed similar efficacy, they were not able to do so in this short time frame. As other products such as malathion, benzyl alcohol $5 \%$ and spinosad $0.9 \%$ have not been established as safe for children below 6 years, the active chemical ingredient in the alternative medicinal pediculicide is a cause for concern [4]. Considering that it is being used on children, and possibly kept on for 8 hours or more as recommended, there is a need to determine the quality and safety of this and other alternative medicinal pediculicide and ensure better control and surveillance of non-prescription preparations used on children.

The second and more positive finding, from this study, is the higher efficacy of herbal preparations. Recommendation of Godapara in Ayurveda does not include pediculosis. It is possible that the plant extracts have several mechanisms of action including their physical properties - thick and sticky nature which causes blocking of respiratory spiracles in lice, and/or the chemical composition which may consist of naturally occurring insecticides meant for the plants' own safety [4,5]. Further scientific study would not only help determine the mechanism of pediculicidal action of the two plants, but also an opportunity to test for safety, toxicity, and allergenic properties of the natural substances in its crude as well as extracted forms before recommending them for use on children [4]. This could prove useful for development of efficient and safe herbal/home remedies that can be used on young 
children to effectively control pediculosis. This being an in vitro study we were unable to evaluate safety.

\section{Acknowledgement}

We wish to thank Dr. DMDEA Gunawardene, Dr. CJ Navaratne, Dr. AOLD Silva, Silva WSA and Mrs.GG De Silva for their technical assistance in laboratory work and Dr.UM Gunasekara for administrative support.

\section{Conflicts of interest}

There are no conflicts of interest.

\section{References}

1. Mumcuoglu KY, Klaus S, Kafka D, Teiler M, Miller J. Clinical observations related to head lice infestation $J$ Am Acad Dermatol 1991; 25: 248-51.

2. Meinking TL, Serrano L, Hard B, et al. Comparative in vitro pediculicidal efficacy of treatments in a resistant head lice population in the United States Arch Dermatol 2002; 138: 220-4.

3. Loomis D, Guyton $\mathrm{K}$, Grosse $\mathrm{Y}$, et al. Carcinogenicity of lindane, DDT, and 2,4dichlorophenoxyacetic acid Lancet Oncol 2015; 16: 891-2.

4. Devore CD, Schutze GE. Head lice Pediatrics 2015; 135: e1355-65.

5. Pearlman DL. A simple treatment for head lice: dry-on, suffocation-based pediculicide Pediatrics 2004; 114: e275-9.

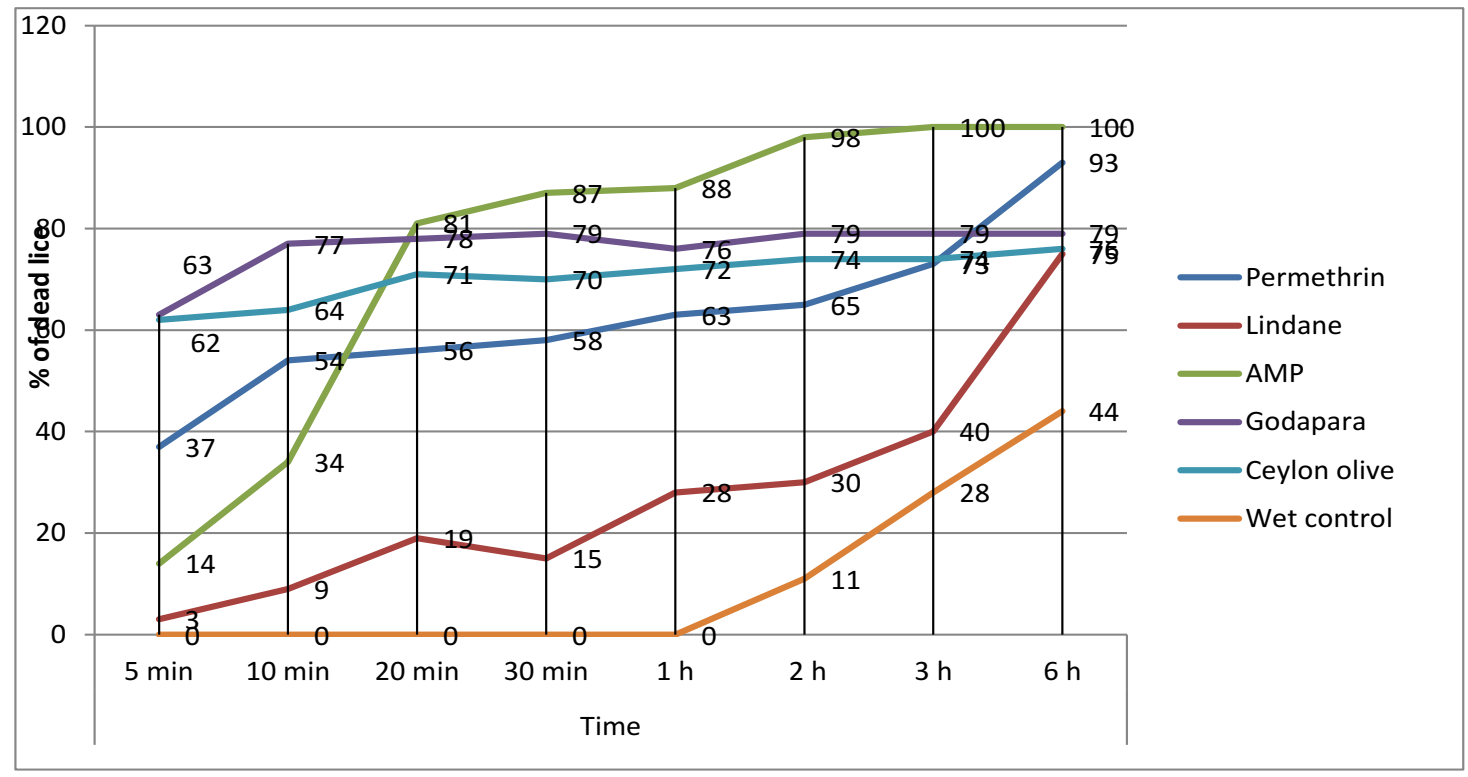

AMP-alternative medicinal pediculicides

Figure 1. Comparison of efficacy of pediculicides against head lice adults and nits 
Table 1. Comparison of efficacy of pediculicides against head lice viable nits (eggs)

\begin{tabular}{|c|c|c|c|c|c|}
\hline \multirow[t]{2}{*}{ Pediculicide } & \multicolumn{5}{|c|}{ Number of hatched nits/nymphs observed after 2 weeks } \\
\hline & Test $1(n=10)$ & Test $2(n=10)$ & Test $3(n=10)$ & Test $4(n=10)$ & $\begin{array}{l}\text { Total tested } \\
(n=40)\end{array}$ \\
\hline Permethrin & 1 & 1 & 0 & 0 & 2 \\
\hline Lindane & 1 & 1 & 1 & 0 & 3 \\
\hline Alternative & 0 & 0 & 0 & 0 & 0 \\
\hline \multicolumn{6}{|l|}{ medicinal } \\
\hline \multicolumn{6}{|l|}{ pediculicide } \\
\hline Wet control & 2 & 2 & 2 & 3 & 9 \\
\hline Dry control & 4 & 2 & 3 & 7 & 16 \\
\hline
\end{tabular}
License, which permits unrestricted use, distribution, and reproduction in any medium, provided the original author and source are credited. 